\title{
Reproducibility of Occurrence of Paroxysmal Atrial Fibrillation in Patients Who Had Acetylcholine Testing
}

\author{
Shozo Sueda ${ }^{1}$ and Tomoki Sakaue ${ }^{2}$
}

\begin{abstract}
Objectives: Paroxysmal atrial fibrillation (PAF) is observed in some certain patients with acetylcholine (ACh) testing. However, it is uncertain for the reproducibility of ACh-induced PAF. We investigated the reproducibility of occurrence of ACh-induced PAF. Methods: We retrospectively investigated the reproducibility of occurrence of PAF in 70 patients who had ACh testing two times apart from at last one month. Incremental intracoronary injection of ACh into the right coronary artery was 20,50 , and $80 \mu \mathrm{g}$, while incremental dose of ACh into the left coronary artery was $20,50,100$, and $200 \mu \mathrm{g}$. Positive provoked spasm was defined as $>90 \%$ transient narrowing with ischemic ECG changes or chest symptoms. Results: Among 70 patients, concordance was observed in 59 patients including 50 patients with both sinus rhythm and 9 patients with both positive PAF. The remaining 11 patients had discordance between the first and the second ACh tests. There were no clinical and angiographical differences except organic stenosis between the first and second ACh testing. All 70 patients had neither past PAF nor future PAF. The rate of coincidence was $84 \%$, while discordance was $16 \%$. AChinducible PAF was reproduced in $45 \%$ of patients who had two times of ACh testing, while the remaining $55 \%$ of patients revealed the discordance of ACh-inducible PAF. Conclusions: Less than half patients disclosed the reproducibility of ACh-inducible PAF.
\end{abstract}

KEY WORDS: acetylcholine, acetylcholine-induced paroxysmal atrial fibrillation, paroxysmal atrial fibrillation, vagal stimulation

\section{Introduction}

In 1986, intracoronary acetylcholine (ACh) testing was first reported $^{1)}$. Since then, intracoronary ACh test has become popular as a spasm provocation test as well as ergonovine (ER) test in the world ${ }^{2-6)}$. Intracoronary injection of ACh has a short half life of this agent and so intracoronary ACh testing considered to be a relatively safe method. However, we experienced some complications such as ventricular fibrillation or tachycardia necessary for electric cardioversion, severe hypotension or left main trunk equivalent spasm during the ACh tests. Furthermore, we also experienced transient paroxysmal atrial fibrillation (PAF) in a sixth of patients who underwent ACh testing based on the Japanese Circulation Society guidelines ${ }^{7,8)}$. ACh-inducible PAF is one of a mechanism of a vagally-mediated $\mathrm{PAF}^{7)}$. There are no reports

${ }^{1}$ Department of Cardiology, Ehime Prefectural Niihama Hospital, Hongou 3 choume 1-1, Niihama City, Ehime Prefecture 792-0042, Japan, ${ }^{2}$ Department of Cardiology, Yawatahama City General Hospital

E-mail: EZF03146@nifty.com

Received: October 1, 2021; Accepted: November 18, 2021

doi: 10.7793/jcad.27.21-00016

(c) The Japanese Coronary Association concerning the reproducibility of ACh-inducible PAF in the same patients. In this article, we retrospectively investigated the reproducibility of ACh-inducible PAF in the same patients.

\section{Methods}

\section{Study patients}

From January 1991 to February 2019, we performed total 1,864 ACh spasm provocation tests. During these periods, we tried to perform the selective spasm provocation tests to examine the incidence of provoked spasm in patients who had undergone coronary angiography whenever possible. Among 1,864 patients with ACh testing, we enrolled 70 patients who had at least two times ACh tests apart from more than one month. As shown in Table 1, female was observed in 10 patients (14\%) and mean age was 64 years old. Ischemic heart disease (IHD) was observed in all 70 patients including 48 patients with angina pectoris, 9 patients with myocardial infarction, and 13 patients with post percutaneous coronary intervention. Organic stenosis $(>75 \%)$ was recognized in 38 patients $(54 \%)$, while 60 patients $(86 \%)$ had a history of smoking. Hypertension was observed in 38 patients (54\%), whereas 42 patients $(60 \%)$ had dyslipidemia. Diabetes mellitus was found in 23 patients (33\%). Subjects were excluded and the provocation test was not performed if patients had left 
Table 1 All patient clinical characteristics

\begin{tabular}{|c|c|c|c|c|c|}
\hline & All patients & Both negative PAF & Both positive PAF & Concordance & Discordance \\
\hline Number & 70 & 50 & 9 & 59 & 11 \\
\hline Sex (female) & $10(14)$ & $6(12)$ & $1(11)$ & $7(12)$ & $3(27)$ \\
\hline Age, $y$, mean \pm SD & $64 \pm 11$ & $64 \pm 11$ & $60 \pm 13$ & $64 \pm 11$ & $64 \pm 9$ \\
\hline Organic stenosis & $38(54)$ & $28(56)$ & $7(78)$ & $35(59)$ & $3(27)$ \\
\hline Follow-up duration (month) & $54 \pm 34$ & $52 \pm 31$ & $44 \pm 37$ & $51 \pm 32$ & $71 \pm 43$ \\
\hline Angina pectoris & $48(69)$ & $38(76)$ & $5(56)$ & $43(73)$ & $5(45)$ \\
\hline Myocardial infarction & $9(13)$ & $5(10)$ & $3(33)$ & $8(14)$ & $1(9)$ \\
\hline $\begin{array}{l}\text { Post percutaneous coronary } \\
\text { intervention }\end{array}$ & $13(19)$ & $7(14)$ & $1(11)$ & $8(14)$ & $5(45)^{*}$ \\
\hline LVEF by UCG $(\%)$ mean \pm SD & $65 \pm 9$ & $66 \pm 9$ & $63 \pm 10$ & $65 \pm 9$ & $62 \pm 11$ \\
\hline \multicolumn{6}{|l|}{ Coronary risk factors } \\
\hline Hypertension & $38(54)$ & $28(56)$ & $4(44)$ & $32(54)$ & $6(55)$ \\
\hline Dyslipidemia & $42(60)$ & $27(54)$ & $6(67)$ & $33(56)$ & $9(82)$ \\
\hline Diabetes mellitus & $23(33)$ & $15(30)$ & $2(22)$ & $17(29)$ & $6(55)$ \\
\hline History of smoking & $60(86)$ & $45(90)$ & $7(78)$ & $52(88)$ & $8(73)$ \\
\hline \multicolumn{6}{|l|}{ Medications before ACh testing } \\
\hline Calcium channel blocker & $50(71)$ & $36(72)$ & $6(67)$ & $42(71)$ & $8(73)$ \\
\hline Dihydropyridine $r$ & $40(57)$ & $27(54)$ & $6(67)$ & $33(56)$ & $7(64)$ \\
\hline Benzodiazepine & $22(31)$ & $18(36)$ & $1(11)$ & $19(32)$ & $3(27)$ \\
\hline Both calcium channel blocker & $12(17)$ & $9(18)$ & $1(11)$ & $10(17)$ & $2(18)$ \\
\hline Nitrates or nicorandil & $40(57)$ & $29(58)$ & $6(67)$ & $35(59)$ & $5(45)$ \\
\hline Beta blocker & $6(9)$ & $3(6)$ & $2(22)$ & $5(8)$ & $1(9)$ \\
\hline ACEI or ARB & $12(17)$ & $7(14)$ & $4(44)$ & $11(19)$ & $1(9)$ \\
\hline Statin & $19(27)$ & $12(24)$ & $3(33)$ & $15(25)$ & $4(36)$ \\
\hline Antiplatelet & $42(60)$ & $27(54)$ & $6(67)$ & $33(56)$ & $9(82)$ \\
\hline
\end{tabular}

ACh: acetylcholine, LVEF: left ventricular ejection fraction, UCG: ultrasound cardiography, ARB: angiotensin receptor blocker, ACEI: angiotensin converting enzyme inhibitor, PAF: paroxysmal atrial fibrillation, *: $p<0.05$ vs. concordance

main narrowing (>50\%), triple-vessel disease, two-vessel disease with total occlusion, heart failure (New York Heart Association functional class III or IV), renal failure (creatinine $>2.0 \mathrm{mg} / \mathrm{dl}$ ), if spontaneous spasm was observed or if isosorbide dinitrate was initially used to relieve spasm in the coronary artery tested.

\section{The definition of PAF}

We defined positive PAF as at least 30 seconds' duration of $\mathrm{PAF}$, and also defined spontaneous relief of ACh-induced PAF as $<15$ minutes' duration. When ACh-induced PAF continues for $>$ 15 minutes, $50 \mathrm{mg}$ of disopyramide or cibenzoline $70 \mathrm{mg}$ solved in warmed $0.9 \%$ saline solution was administered intravenously for 5 minutes.

\section{The definition of positive spasm and spasm configuration}

Generally, we defined positive spasm as $\geq 90 \%$ transient stenosis and usual chest symptom or ischemic ECG changes. The degree of ST-segment depression was measured $80 \mathrm{msec}$ after the $\mathrm{J}$ point. We considered a result to be positive when at least 1 of the following ischemic ECG changes was demonstrated during and/or after the ACh test: (1) ST-segment elevation of $\geq$ $0.1 \mathrm{mV}$ in at least 2 contiguous leads ; (2) ST-segment depression of $0.1 \mathrm{mV}$ in at least 2 contiguous leads. We also considered negative $\mathrm{U}$ wave as positive ischemic ECG change. Focal spasm was defined as a discrete transient vessel narrowing $\geq 90 \%$ localized in a major coronary artery, whereas diffuse spasm was diag- nosed when transient vessel narrowing $\geq 90 \%$, compared with baseline coronary angiography, was observed in $\geq 2$ adjacent coronary segments of epicardial coronary arteries.

\section{Spasm provocation test}

All drugs except for nitroglycerine were discontinued for $\geq 24$ hours before the study, and nitroglycerine was also discontinued $\geq 4$ hours before the study. Cardiac catheterization was performed from 9: $00 \mathrm{am}$ to 4: $00 \mathrm{pm}$ in the fasting state. After control coronary arteriograms of the left coronary artery (LCA) in the right anterior oblique with caudal projection and of the right coronary artery (RCA) in the left anterior oblique with cranial projection were obtained by injection of $8-10 \mathrm{ml}$ of contrast medium, a temporary pacemaker was inserted into the right ventricle of each ACh testing patient and the pacing rate was set at 40 45 beats/min.

Provocation of coronary artery spasm was performed with an intracoronary injection of ACh, as previously reported ${ }^{9,10)}$. ACh chloride was injected in incremental doses of 20,50 and $80 \mu \mathrm{g}$ into the RCA and of 20,50, 100 and $200 \mu \mathrm{g}$ into the LCA over 20 seconds with at least a 3-minute interval between each injection. Coronary arteriography was performed when ST-segment changes and/or, chest pain occurred or 1-2 minutes after the completion of each injection. When an induced coronary spasm did not resolve spontaneously within 3 minutes after the comple- 
Journal of Coronary Artery Disease 2021; 27: 105-111

Table 2 Clinical and angiographical findings in patients with both PAF positive

\begin{tabular}{|c|c|c|c|c|c|c|c|c|c|}
\hline \multirow{2}{*}{ No } & \multirow{2}{*}{ Age } & \multirow{2}{*}{ Sex } & \multirow{2}{*}{ Diagnosis } & \multicolumn{3}{|c|}{ First ACh test } & \multicolumn{3}{|c|}{ Second ACh test } \\
\hline & & & & ACh dose & Spasm & PAF & ACh dose & Spasm & PAF \\
\hline \multirow[t]{2}{*}{1} & 56 & M & Rest AP & RCA (50) & $1(\mathrm{~d})$ & Disopyramide $50 \mathrm{mg}$ & RCA (50) & No spasm & Natural \\
\hline & & & & LCA $(10 / 20)$ & $7(f)$ & & LCA $(\mathbf{5 0 / 1 0 0})$ & $8(t)$ & Natural \\
\hline \multirow[t]{2}{*}{2} & 47 & M & Effort AP & RCA $(\mathbf{2 0} / \mathbf{5 0})$ & $2(d)$ & Disopyramide $50 \mathrm{mg}$ & $\operatorname{RCA}(\mathbf{5 0} / \mathbf{8 0})$ & No spasm & 50 Natural \\
\hline & & & & LCA $(50)$ & $7(f)$ & & LCA $(50 / 100)$ & No spasm & 80 disopyramide $50 \mathrm{mg}$ \\
\hline \multirow[t]{2}{*}{3} & 62 & $\mathrm{~F}$ & MI & $\mathrm{RCA}(\mathbf{8 0})$ & No spasm & Disopyramide $50 \mathrm{mg}$ & $\operatorname{RCA}(\mathbf{5 0} / 80)$ & No spasm & Disopyramide $50 \mathrm{mg}$ \\
\hline & & & & $\operatorname{LCA}(50 / 100)$ & $8(\mathrm{~d})$ & & $\operatorname{LCA}(50 / 100$ & No spasm & \\
\hline \multirow[t]{2}{*}{4} & 64 & M & MI & RCA $(20 / 50 / 80)$ & $1(\mathrm{~d})$ & Disopyramide $50 \mathrm{mg}$ & RCA $(20 / 50 / 80)$ & $3(\mathrm{t})$ & Natural \\
\hline & & & & $\operatorname{LCA}(50 / 100)$ & $6(d) 11(d)$ & & $\operatorname{LCA}(50)$ & $7(\mathrm{t})$ & \\
\hline \multirow[t]{2}{*}{5} & 60 & M & Rest AP & $\operatorname{RCA}(25 / \mathbf{5 0})$ & No spasm & Cibenzoline $70 \mathrm{mg}$ & $\operatorname{RCA}(\mathbf{5 0})$ & No spasm & Cibenzoline $70 \mathrm{mg}$ \\
\hline & & & & LCA $(25 / 50)$ & $7(\mathrm{~d})$ & & LCA $(25 / 50 / 100)$ & No spasm & \\
\hline \multirow[t]{2}{*}{6} & 39 & M & MI & $\operatorname{RCA}(\mathbf{5 0})$ & No spasm & Natural & RCA (50) & $4(d)$ & Natural \\
\hline & & & & LCA $(50 / 100)$ & $7(\mathrm{t})$ & & LCA $(25 / 50)$ & $7(d)$ & \\
\hline \multirow[t]{2}{*}{7} & 72 & M & Effort AP & RCA $(25 / 50)$ & $4(d)$ & Natural & $\mathrm{RCA}(\mathbf{7 5})$ & $4(d)$ & Cibenzoline $70 \mathrm{mg}$ \\
\hline & & & & LCA $(25 / 50 / 100)$ & $8(d) 13(t)$ & & LCA $(25 / 50 / 100 / 200)$ & $8(d) 11(d)$ & \\
\hline \multirow[t]{2}{*}{8} & 63 & M & Post P & $\operatorname{RCA}(\mathbf{5 0} / 80)$ & No spasm & Cibenzoline $70 \mathrm{mg}$ & $\operatorname{RCA}(\mathbf{5 0})$ & No spasm & Natural \\
\hline & & & & LCA $(20 / 50 / 100)$ & $8(d)$ & & $\operatorname{LCA}(25 / \mathbf{5 0} / \mathbf{1 0 0})$ & No spasm & Natural \\
\hline \multirow[t]{2}{*}{9} & 85 & M & Post P & $\operatorname{RCA}(25 / 50 / 75)$ & No spasm & Cibenzoline $70 \mathrm{mg}$ & $\mathrm{RCA}(\mathbf{8 0})$ & No spasm & Cibenzoline $70 \mathrm{mg}$ \\
\hline & & & & $\operatorname{LCA}(25 / 50 / 100 / 200)$ & & & LCA (100) & No spasm & \\
\hline
\end{tabular}

ACh: acetylcholine, AP: angina pectoris, MI: myocardial infarction, Post P: post percutaneous coronary intervention, RCA: right coronary artery, LCA: left coronary artery, (d): diffuse spasm, (f): focal spasm, (t): total spasm, PAF: paroxysmal atrial fibrillation, bold number: PAF induced dose of $\mathrm{ACh}$

tion of ACh injections or when hemodynamic instability occurred as the result of coronary spasm, 2.5 to $5.0 \mathrm{mg}$ of nitrate was injected into the involved vessel. A standard 12-lead electrocardiogram was recorded every 30 seconds. We used the ECG findings when $\mathrm{ACh}$, saline and contrast medium were not injected into the responsible vessels for at least 60 seconds. After the spasm provocation tests were completed, an intracoronary injection of $5.0 \mathrm{mg}$ isosorbide dinitrate was administered, and coronary arteriography was then performed in multiple projections.

During the study, arterial blood pressure and ECG were continuously monitored on an oscilloscope by Nihon-Kohden polygraphy (Tokyo, Japan). Patients with catheter-induced spasms were excluded from this study. Significant organic stenosis was defined as $>75$ percent luminal narrowing according to the American College of Cardiology/American Heart Association classification $^{11)}$. We defined hypoplastic RCA as a very small RCA when the RCA supplies only to the right ventricle and ends before reaching the crux of the heart and prior to splitting off the posterior descending artery and posterolateral artery. We did not perform ACh testing in the hypoplastic $\mathrm{RCA}^{12)}$.

The study protocol complied with the Declaration of Helsinki. Written informed consent was obtained from all patients before performing the pharmacological spasm provocation tests and the protocol of this study was in agreement with the guidelines of the ethical committee at our institution.

\section{Statistical analysis}

Data analysis was carried out with SPSS (version 22.0, IBM Japan, Ltd., Tokyo, Japan). All data were presented as the mean \pm 1 SD. Clinical characteristics among three groups and angiographical findings and medications between the first and second tests were analyzed by the Fisher's exact test with correction or the Mann-Whitney test. $\mathrm{P}<0.05$ was considered significant.

\section{Results}

During the follow-up periods $(64 \pm 11$ months), we found no previous PAF and no future PAF in all 70 patients. According to the response of ACh testing, we classified 70 patients into the three groups: 50 patients with both sinus rhythm, 9 patients with both positive PAF, and 11 patients with either of one PAF positive. We could not perform ACh tests in 5 RCAs (hypoplastic RCA: 2 vessels, obstructive stenosis $>90 \%$ : 2 vessels, after the administration of nitroglycerine into the LCA: 1 vessel) and one LCA (after the administration of nitroglycerine into the RCA) of the first ACh testing, whereas we also could not perform these tests in 2 RCAs (hypoplastic RCA) and one LCA (after the administration of nitroglycerine into the RCA) of the second $\mathrm{ACh}$ tests. We could perform both times and both coronary ACh testing in 63 patients (90\%) of the study subjects.

Concordance of PAF or sinus rhythm

As shown in Table 1, 59 patients had the same response be- 
Journal of Coronary Artery Disease 2021; 27: 105-111

Table 3 Clinical and angiographical findings in patients with discordance PAF positive

\begin{tabular}{|c|c|c|c|c|c|c|c|c|c|}
\hline \multirow{2}{*}{ No } & \multirow{2}{*}{ Age } & \multirow{2}{*}{ Sex } & \multirow{2}{*}{ Diagnosis } & \multicolumn{3}{|c|}{ First ACh test } & \multicolumn{3}{|c|}{ Second ACh test } \\
\hline & & & & ACh dose & Spasm & PAF & ACh dose & Spasm & PAF \\
\hline \multirow[t]{2}{*}{1} & 54 & M & Post P & $\operatorname{RCA}(\mathbf{5 0} / 80)$ & No spasm & Disopyramide $50 \mathrm{mg}$ & RCA $(50 / 80)$ & No spasm & $(-)$ \\
\hline & & & & LCA $(\mathbf{5 0 / 1 0 0})$ & No spasm & Natural & $\operatorname{LCA}(50 / 100)$ & No spasm & $(-)$ \\
\hline \multirow[t]{2}{*}{2} & 71 & M & $\mathrm{R} \& \mathrm{E} A \mathrm{~A}$ & RCA $(50)$ & $4(\mathrm{~d})$ & & $\mathrm{RCA}(50)$ & No spasm & $(-)$ \\
\hline & & & & LCA $(\mathbf{1 0 0})$ & $8(d)$ & Natural & LCA $(50 / 100)$ & $8(d)$ & $(-)$ \\
\hline \multirow[t]{2}{*}{3} & 65 & $\mathrm{~F}$ & Post P & RCA $(\mathbf{2 0} / 50 / 80)$ & No spasm & Natural & $\operatorname{RCA}(80)$ & No spasm & $(-)$ \\
\hline & & & & LCA $(20 / 50 / 100)$ & No spasm & & LCA $(50 / 100 / 20)$ & No spasm & $(-)$ \\
\hline \multirow[t]{2}{*}{4} & 72 & M & MI & RCA (20/50) & $2(d)$ & & RCA $(20 / 50 / 80)$ & No spasm & $(-)$ \\
\hline & & & & LCA $(\mathbf{1 0 0})$ & $8(\mathrm{~d}) 11(\mathrm{f})$ & Natural & $\operatorname{LCA}(50 / 100 / 200)$ & $8(t) 13(d)$ & $(-)$ \\
\hline \multirow[t]{2}{*}{5} & 69 & $\mathrm{~F}$ & Rest AP & $\operatorname{RCA}(\mathbf{2 0} / \mathbf{5 0})$ & $2(t)$ & Natural & RCA (80) & No spasm & $(-)$ \\
\hline & & & & LCA $(20 / 50 / 100 / 200)$ & No spasm & & LCA $(20 / 100 / 200)$ & No spasm & $(-)$ \\
\hline \multirow[t]{2}{*}{6} & 73 & M & Post P & $\operatorname{RCA}(\mathbf{5 0} / 80)$ & $4(d)$ & Disopyramide $50 \mathrm{mg}$ & RCA $(25 / 50)$ & No spasm & $(-)$ \\
\hline & & & & LCA $(25 / 50 / 100)$ & $7(d)$ & & LCA $(50 / 100)$ & Spastic & $(-)$ \\
\hline \multirow[t]{2}{*}{7} & 45 & M & Rest AP & RCA $(20 / 50 / 80)$ & 3(f) & Natural & $\mathrm{RCA}(80)$ & $4(d)$ & $(-)$ \\
\hline & & & & LCA $(20 / 50 / 100)$ & & & $\begin{array}{l}\text { LCA } \\
(20 / 50 / 100 / 200)\end{array}$ & $7(d)$ & $(-)$ \\
\hline \multirow[t]{2}{*}{8} & 66 & $\mathrm{~F}$ & Post P & RCA (50/80) & No spasm & $(-)$ & $\operatorname{RCA}(50 / 80)$ & No spasm & Disopyramide $50 \mathrm{mg}$ \\
\hline & & & & LCA $(50 / 100)$ & No spasm & $(-)$ & LCA $(50 / 100)$ & No spasm & \\
\hline \multirow[t]{2}{*}{9} & 69 & M & Post P & RCA $(25 / 50$ & No spasm & $(-)$ & RCA (75) & No spasm & Natural \\
\hline & & & & LCA $(25 / 50 / 100)$ & $7(\mathrm{~d})$ & $(-)$ & LCA $(50 / 100)$ & $7(\mathrm{~d})$ & \\
\hline \multirow[t]{2}{*}{10} & 54 & M & MI & $\mathrm{RCA}(20)$ & $2(\mathrm{~d})$ & $(-)$ & $\operatorname{RCA}(\mathbf{2 0} / 50)$ & $4(d)$ & Natural \\
\hline & & & & LCA $(20 / 50 / 100)$ & $7(\mathrm{t}) 13(\mathrm{f})$ & $(-)$ & LCA $(20 / 50 / 100)$ & $7(d)$ & \\
\hline \multirow[t]{2}{*}{11} & 65 & M & $\mathrm{R} \& \mathrm{E} A \mathrm{AP}$ & RCA $(20 / 50)$ & $1(\mathrm{~d})$ & $(-)$ & RCA (50) & $4(\mathrm{~d})$ & \\
\hline & & & & LCA $(50 / 100)$ & $6(d) 11(d)$ & $(-)$ & LCA $(\mathbf{1 0 0})$ & $6(f) 11(f)$ & $\begin{array}{l}\text { Disopyramide } 50 \mathrm{mg} \\
\text { Etilefrine } 2 \mathrm{mg}\end{array}$ \\
\hline
\end{tabular}

ACh: acetylcholine, AP: angina pectoris, MI: myocardial infarction, Post P: post percutaneous coronary intervention, RCA: right coronary artery, LCA: left coronary artery, (d): diffuse spasm, (f): focal spasm, (t): total spasm, PAF: paroxysmal atrial fibrillation, bold number: PAF induced ACh dose, under PAF test

tween the two ACh testing. There was no clinical difference between both sinus rhythm and both positive PAF. Table 2 showed the clinical and angiographical findings in patients with both occurrence of PAF. PAF was observed in all 9 patients in the first RCA ACh testing, whereas, PAF was observed in all 9 RCA ACh testing and two LCA ACh tests in the second ACh test. Positive spasm in 59 patients with concordance groups was observed in 49 patients $(83 \%)$ in the first ACh testing, while positive spasm in the second ACh test was recognized in 41 patients (69\%). In 9 patients with both PAF, positive spasm of first ACh testing was observed in 8 patients ( $89 \%$ ), whereas positive spasm in the second ACh test was found in 4 patients (44\%).

\section{Comparisons of concordance and discordance PAF}

Discordance occurrence of PAF between the two ACh tests was found in 11 patients, as shown in Table 3. In the first ACh testing, 7 patients had PAF including 4 RCA ACh tests, 2 LCA ACh tests and one both ACh test, while 4 patients had PAF including 3 RCA ACh tests and one LCA ACh test in the second ACh testing. Positive spasm was found in 7 patients (64\%) in the first ACh testing, whereas positive spasm in the second ACh test was revealed in 6 patients $(55 \%)$. However, there were no clinical differences between the concordance group and discordance group as shown in Table 1.

\section{Comparisons between first and second ACh testing}

As shown in Table 4, the frequency of organic stenosis in second ACh testing was significantly lower than that in first $\mathrm{ACh}$ tests. However, there was no differences concerning the provoked spasm and medications between the two ACh testing.

\section{Complications of ACh testing}

Left amin trunk equivalent spasm was observed in just one patient. However, no irreversible complications were observed in this study.

\section{Discussion}

In this article, we found no correlation of reproducibility of PAF between first ACh testing and second ACh tests. Discordance of ACh-inducible PAF was observed in $14 \%$ of patients who had two times ACh tests apart from more than one month. Furthermore, during the follow-up periods (64 \pm 11 months), we found no previous PAF and no future PAF in these 70 patients. 
Journal of Coronary Artery Disease 2021; 27: 105-111

Table 4 Angiographical findings and medications before acetylcholine tests

\begin{tabular}{lccc}
\hline & First ACh testing & Second ACh testing & p value \\
\hline Number & 70 & 70 & \\
Organic stenosis & $38(54)$ & $16(23)$ & $<0.001$ \\
Right coronary artery & $13(19)$ & $6(9)$ & 0.1387 \\
Left circumflex coronary artery & $19(27)$ & $3(4)$ & $<0.001$ \\
Left anterior descending coronary artery & $48(69)$ & $8(11)$ & $<0.001$ \\
1 vessel disease & $33(47)$ & $15(21)$ & $<0.01$ \\
2 vessel diseases & $5(7)$ & $1(1)$ & 0.2106 \\
Provoked positive spasm & $54(77)$ & $46(66)$ & 0.1344 \\
Right coronary artery & $36(51)$ & $32(46)$ & 0.4987 \\
Left circumflex coronary artery & $17(24)$ & $13(19)$ & 0.4100 \\
Left anterior descending coronary artery & $45(64)$ & $37(53)$ & 0.1698 \\
1 vessel spasm & $23(33)$ & $19(27)$ & 0.4606 \\
2 vessel spasm & $19(27)$ & $19(27)$ & 1.0000 \\
3 vessel spasm & $12(17)$ & $8(11)$ & 0.3339 \\
Medications before ACh testing & & & \\
Calcium channel blocker & $50(71)$ & $57(81)$ & 0.1633 \\
Dihydropyridine r & $40(57)$ & $43(61)$ & 0.6058 \\
Benzodiazepine & $22(31)$ & $21(30)$ & 0.8546 \\
Both calcium channel blocker & $12(17)$ & $7(10)$ & 0.2172 \\
Nitrates or nicorandil & $40(57)$ & $44(63)$ & 0.4901 \\
Beta blocker & $6(9)$ & $3(4)$ & 0.4907 \\
ACEI or ARB & $10(14)$ & $12(17)$ & 0.6423 \\
Statin & $19(27)$ & $20(29)$ & 0.8504 \\
Antiplatelet & $42(60)$ & $44(63)$ & 0.7284 \\
\hline A & & &
\end{tabular}

ACh: acetylcholine, ARB: angiotensin receptor blocker, ACEI: angiotensin converting enzyme inhibitor

This may suggest that ACh-inducible PAF is a reaction of individual response to just $\mathrm{ACh}$ at that time. The clinical implications of ACh-inducible PAF may have limitations of estimating previous and future occurrence of PAF.

\section{ACh-inducible PAF in Western and Japanese populations}

In the past report of ours, we had no correlation between the daily PAF and ACh-inducible $\mathrm{PAF}^{7)}$. Among 717 patients without persistent atrial fibrillation, we experienced transient PAF during ACh testing in 116 patients (16.2\%). Daily PAF in patients with ACh-induced PAF was not different from that in those with ACh-induced no PAF (2.6\% (3/116) vs. 1.0\% (6/601), ns). However, Saito et al. reported that a history of PAF and body mass index were independent predictors for occurrence of PAF during intracoronary $\mathrm{ACh}$ provocation test by multivariate logistic regression analysis ${ }^{13)}$. PAF during ACh testing was observed in just 8\% (31 patients) among 377 patients without persistent atrial fibrillation who underwent intracoronary ACh provocation tests. The occurrence rate of PAF during ACh testing was lower than the data of previous ours. Maximum ACh dose of $80 \mu \mathrm{g}$ into the RCA and $200 \mu \mathrm{g}$ into the LCA of our study might be concerned. They employed maximum ACh dose of $50 \mu \mathrm{g}$ into the RCA and $100 \mu \mathrm{g}$ into the LCA. In contrast, in a Western report, one patient had fast PAF that resolved spontaneously after discontinuing the ACh injection ${ }^{14}$. The observation of short phases of PAF during ACh testing was not uncommon, but was not registered as a complication in their study. Incremental doses of 2, 20,100 , and $200 \mu \mathrm{g}$ of ACh were manually infused over a period of 3 minutes into the LCA without a pacemaker. If no proved spasm in the LCA was revealed, $80 \mu \mathrm{g}$ of ACh was injected into the RCA. However, intracoronary RCA ACh testing was performed in just 34.4\% (291/847) of their study. Accurate occurrence of PAF in their study was obscure. In another Caucasian report, PAF is a rare complication with ACh infusion over 1 minute into the LCA $(1.1 \%)$. While $6.9 \%$ of patients developed atrioventricular block and 3.3\% developed significant bradycardia, these conditions readily reversed once the administration of ACh was stopped ${ }^{15)}$. Western cardiologists administered intracoronary ACh over 1-3 minutes without a pacemaker, whereas we injected intracoronary ACh within 20 seconds under pacemaker. Intracoronary RCA ACh testing without a pacemaker is difficult to perform possibly due to bradycardia and block. According to our previous report, PAF was observed in 109 patients (94\%) in the RCA ACh testing, whereas 9 patients $(8 \%)$ had PAF in the LCA ACh tests ${ }^{7)}$. Two patients (2\%) had PAF on both coronary arteries. In this study, PAF was recognized in 26 RCAs, while 6 LCAs had PAF. PAF was observed in 3 patients on both coronary arteries (one patient with discordance PAF of first ACh test \& two patients with concordance PAF of second ACh tests). 


\section{Clinical implications of ACh-inducible PAF}

The majority of ACh-inducible PAF was observed in the RCA ACh testing. However, the occurrence of ACh-inducible PAF in Western reports was unclear. Furthermore, we could not find the reproducibility of ACh-inducible PAF in the previous reports. This is the first report regarding the reproducibility of ACh-inducible PAF. In this study, we could perform both times and both coronary ACh testing in $90 \%$ of study populations. The administration time of ACh may contribute to the occurrence of PAF and vagally mediated PAF is a mechanism of ACh-inducible PAF. In this study, a previous history of PAF or future PAF in patients with ACh-induced PAF was not found. We suggest that ACh-induced PAF may react the individual response of ACh in the cardiac catheterization laboratory at that time. Mechanism favoring the occurrence of PAF are complex and the occurrence of PAF greatly depends on variation of the autonomic tone, with a primary increase in adrenergic tone followed by an abrupt shift toward vagal predominance ${ }^{16,17)}$. It may be difficult to estimate the occurrence of clinical daily PAF or persistent atrial fibrillation in the future by intracoronary ACh-inducible PAF alone. Further research is necessary to clarify the reproducibility of the occurrence of ACh-inducible PAF in Western and Japanese subjects.

\section{Limitations}

Several limitations were recognized in this study. First is a single center, retrospective, small study sample and not prospective study. Second, we could not perform Holter monitoring in all patients during follow-up periods at a constant examination. We may miss a transient silent PAF. Third, follow-up duration was short to verify the correlation between ACh-inducible PAF and future PAF or persistent atrial fibrillation. It may be better more-longer duration of follow-up periods than 10 years. Fourth, we could not perform the complete ACh testing on both times. However, we could perform both times and both coronary ACh testing in $90 \%$ of study populations. Further study is necessary for clarifying the correlation between ACh-inducible PAF and future occurrence of PAF or persistent atrial fibrillation.

\section{Conclusions}

There was no correlation regarding the reproducibility between first and second ACh-inducible PAF. This study suggests that it may be difficult to estimate the past and future occurrence of PAF according to the ACh-inducible PAF alone.

\section{Conflicts of interest}

The authors declare that they have no conflict of interest.

\section{Sources of funding}

None.

\section{References}

1) Yasue H, Horio Y, Nakamura N, et al: Induction of coronary artery spasm by acetylcholine in patients with variant angina: possible role of the parasympathetic nervous system in the pathogenesis of coronary artery spasm. Circulation 1986; 74: 955-963

2) Okumura K, Yasue H, Matsuyama K, et al: Sensitivity and specificity of intracoronary injection of acetylcholine for the induction of coronary artery spasm. J Am Coll Cardiol 1988; 12: 883-888

3) Okumura K, Yasue H, Horio Y, et al: Multivessel coronary spasm in patients with variant angina: a study with intracoronary injection of acetylcholine. Circulation 1988; 77: 535-542

4) Sato K, Kaikita K, Nakayama N, et al: Coronary vasomotor response to intracoronary acetylcholine injection, clinical features, and longterm prognosis in 873 consecutive patients with coronary spasm: analysis of a single-center study over 20 years. J Am Heart Assoc 2013; 2: e000227

5) Ong P, Athabasiadis A, Borgulya G, et al: High prevalence of a pathological reponse to acetylcholine testing in patients with stable angina pectoris and unobstructed coronary arteries: the ACOVA study (Abnormal COronary VAsomotion in patients with stable angina and unobstructed coronary arteries). J Am Coll Cardiol 2012; 59: 655-662

6) Zaya M, Mehta PK, Merz CNB: Provocative testing for coronary reactivity and spasm. J Am Coll Cardiol 2014; 63: 103-109

7) Sueda S, Fukuda H, Watanabe K, et al: Clinical characteristics and possible mechanism of paroxysmal atrial fibrillation induced by intracoronary injection of acetylcholine. Am J Cardiol 2001; 88: 570573

8) JCS joint working group: Guidelines for diagnosis and treatment of patients with vasospastic angina (Coronary spastic angina) (JCS 2013). Circ J 2014; 78: 2779-2801

9) Sueda S, Ochi N, Kawada H, et al: Frequency of provoked coronary vasospasm in patients undergoing coronary arteriography with spasm provocation test of acetylcholine. Am J Cardiol 1999; 83: 11861190

10) Sueda S, Saeki H, Otani T, et al: Major complications during spasm provocation tests with an intracoronary injection of acetylcholine. Am J Cardiol 2000; 85: 391-394

11) Scanlon PJ, Faxon DP, Audet AM, et al: ACC/AHA guidelines for coronary angiography: a report of the American College of Cardiology/American Heart Association Task Force on practice guidelines (Committee on Coronary Angiography) developed in collaboration with the Society for Cardiac Angiography and Interventions. J Am Coll Cardiol 1999; 33: 1756-1824

12) Aydar Y, Yazici HU, Birdane A, et al: Relationship between hypoplastic right coronary artery and coronary artery anomalies. Eur Rev Med Pharmacol Sci 2013; 17: 694-700

13) Saito $Y$, Kitahara H, Shoji T, et al: Paroxysmal atrial fibrillation during intracoronary acetylcholine provocation test. Heart Vessels 2017; 32: 902-908

14) Ong P, Athanasiadis A, Borgulya G, et al: Clinical usefulness, angiographic characteristics, and safety evaluation of intracoronary acetylcholine provocation testing among 921 consecutive white patients with unobstructed coronary arteries. Circulation 2014; 129: 1723 


\section{Journal of Coronary Artery Disease 2021; 27: 105-111}

1730

15) Pargaonkar VS, Lee JH, Chow EKH, et al: Dose-response relationship between intracoronary acetylcholine and minimal lumen diameter in coronary endothelial function testing to women and men with angina and no obstructive coronary artery disease. Cir Cardiovasc Interv 2020; 13: e008587
16) Bettoni M, Zimmermann M: Autonomic tone variations before the onset of paroxysmal atrial fibrillation. Circulation 2002; 105: 27532759

17) Amar D, Zhang H, Miodownik S, et al: Competing autonomic mechanisms precede the onset of postoperative atrial fibrillation. J Am Coll Cardiol 2003; 42: 1262-1268 